\title{
3D Fluorescence Characterization of Synthetic Organic Dyes
}

\author{
Leonard J. Soltzberg ${ }^{1}$, Sandy Lor $^{1}$, Nnennaya Okey-Igwe ${ }^{1}$, Richard Newman ${ }^{2}$ \\ ${ }^{1}$ Department of Chemistry \& Physics, Simmons College, Boston, USA \\ ${ }^{2}$ Scientific Research Lab, Museum of Fine Arts, Boston, USA \\ Email: lsoltzberg@simmons.edu
}

Received June 27, 2012; revised August 2, 2012; accepted August 13, 2012

\begin{abstract}
The identification of dyes is important in research on museum artefacts as well as in forensic applications. UV-visible absorption spectroscopy cannot unambiguously distinguish dyes with similar hues, while mass spectrometry may fail to distinguish isobaric dyes. The detailed patterns produced by $3 \mathrm{D}$ fluorescence spectroscopy appear to be virtually unique, even among dyes that are closely related positional isomers. We report these patterns for 65 dyes from the Schweppe Library of Synthetic Organic Dyes as well as measurements suggesting both the capabilities and limitations of this method.
\end{abstract}

Keywords: Fluorescence; Spectrophotometry; Dye Analysis; MALDI-TOF; Mass Spectrometry

\section{Introduction}

The identification of dyes and pigments is important in a museum setting for authentication of artefacts, conservation, and for art historical research. For example, the recent identification of the yellow lichen-derived dye vulpinic acid in a Northwest-Coast ceremonial blanket dictated the need to display this artefact in subdued light [1]. MALDITOF (matrix-assisted laser desorption time-of-flight) mass spectrometry has been used successfully to characterize a library of synthetic organic dyes [2]. However, that method is less useful for distinguishing isomeric dyes such as Acid Yellow 36 (CI 13065) and Acid Orange 5 (CI 13080) because the molecules are isobaric.

3D fluorescence spectra, obtained by measuring fluorescence emission via scanning both the excitation wavelength (EX) and the emission wavelength (EM), can be presented as contour plots on X-Y axes defined by EM and EX. It has been suggested that these fingerprint-like patterns are better able to discriminate among similar dyes than are individual numerical data, such as tabulated absorption maxima [3-5].

Interesting work has been done using fiber optic probe reflectance 3D fluorescence spectrophotometry as applied to dye analysis in situ in textiles, prints and manuscripts [3-5]. While that approach has the advantage of being rigorously non-destructive, the quality of the spectra makes them difficult to apply as an identification tool. In this work, we used 3D fluorescence spectra to characterize water solutions of dyes in an important standard dye collection
[6], supplemented by MALDI-TOF mass spectra. The use of solutions improves the quality of the spectra, eliminates some background artifacts and yet, because of the sensitivity of fluorescence spectrophotometry, requires only nanomole quantities of analyte. This same dye collection has been the subject of analysis by HPLC [4] and MALDITOF mass spectrometry [2].

\section{Experimental}

\subsection{Instrumentation}

We used a Hitachi F4500 fluorescence spectrophotometer and a Hitachi 650-0116 microcell to obtain fluorescence spectra; $200 \mu \mathrm{L}$ of solution was required for this cell. The excitation wavelength EX was scanned from 250 - $380 \mathrm{~nm}$ and the emission wavelength EM was scanned from $395-700 \mathrm{~nm}$. These two ranges purposely do not overlap in order to avoid exposing the photomultiplier to 1 st order scattering from the Xe light source. The scanning rate was $1200 \mathrm{~nm} / \mathrm{min}$, and the response time was set at $0.1 \mathrm{sec}$. These conditions allowed us to obtain a spectrum in less than 8 minutes with no apparent loss of detail, compared with slower scan speeds.

For those dyes for which the extinction coefficients were available, concentrations of the test solutions were determined by measuring the absorbance using an Agilent 8453 diode array UV-visible spectrophotometer and an Agilent 5062-2496 $50 \mu \mathrm{L}$ ultra-micro quartz cell.

MALDI-TOF mass spectra were obtained with a Bruker Omniflex instrument in reflectron mode, using the matrix 
9-aminoacridine and sample preparation methods described elsewhere [2,7].

\subsection{Materials}

The original Schweppe Library of Synthetic Organic Dyes [6] is housed at the J. P. Getty Museum in Los Angeles, CA. The samples from this Library in the possession of the Boston Museum of Fine Arts (MFA) are solutions in methanol. For the current work, we diluted samples from the MFA collection with Sigma-Aldrich HPLC-Plus water (Sigma-Aldrich 34,877) to give concentrations suitable for recording fluorescence spectra.

Since the original Schweppe collection is over 50 years old, we also examined fresh commercial samples of some of the dyes, especially those showing obvious decomposition based on the appearance of the library solutions: Acid Black 1 (CI 20470), Basic Green 4 (CI 42000), Acid Blue 93 (CI 42780), Acid Black 2 (CI 50420), Murexide (CI 56085), Mordant Red 3 (CI 58005), and Acid Blue 74 (CI 73015). For these dyes, the fluorescence spectra shown in Table 1 were obtained using fresh dye samples.

\section{Results and Discussion}

The 3D fluorescence spectra of all 65 Schweppe reference dyes are shown in Table $\mathbf{1}$ along with the corresponding molecular structures, organized by structure type. We refer to those spectra below using the Schweppe number; e.g. "S10".

The concentrations of the dyes in the Schweppe collection are not known. However, for dyes for which we could find the extinction coefficients [8], we checked the concentration of the test solutions used for the fluorescence spectra by measuring their absorption spectra in the range $200-800 \mathrm{~nm}$ with the Agilent diode array spectrometer. These concentrations ranged from $1.5 \times 10^{-6} \mathrm{M}$ to $9.0 \times 10^{-5} \mathrm{M}$.

\subsection{Instrumental Artifacts}

Certain artifacts are characteristic of fluorescence spectra and need to be taken into account when examining these 3D fluorescence spectra. First, Rayleigh scattering of the excitation beam produces a distinct diagonal feature, as seen, for example, with S10 Acid Orange 6 (CI 14270) (Table 1) and with the water blank shown in Figure 1. We purposely chose the excitation and emission wavelength ranges to exclude scattered light from the intense $1^{\text {st }}$ order beam, but the Rayleigh scattering of the $2^{\text {nd }}$ order diffracted light from the Xe source is clearly seen in many of the spectra along the diagonal line $\lambda_{\mathrm{EM}}=2 \lambda_{\mathrm{EX}}$. This feature provides an approximate gauge for the fluorescent intensity of the dye itself. For weakly fluorescent dyes such as S10, the Rayleigh scattering is more prominent than any features arising from the dye. With a strongly fluorescent dye such as S50 Basic Red 1 (CI 45160), the Rayleigh scattering is not even seen, relative to the intensity of the fluorescence of the dye itself.

Another artifact is Raman scattering from the solvent. This phenomenon produces a diagonal feature in which the energy of a scattered photon is shifted by a constant amount from the excitation photon energy. For water, the features arising from Raman scattering appear at the EX/EM values shown in Table 2 and in the pattern at the upper left of Figure 1. The Raman scattering is considerably weaker than the Rayleigh scattering. A different Raman scattering feature arising from the $v_{2}$ vibration of water [9] and excited by the 2 nd order light from the instrument lamp, appears below the Rayleigh scattering feature in Figure $\mathbf{1}$; this feature is seen only in a few of the dye spectra in Table 1.

Finally, it has recently been reported that the fluorescence spectra of highly purified water show features attributed to interaction among trace impurities, including air gases, and the hydrogen bond network of the water [10]. We believe that the feature seen in a number of our dye spectra at $\mathrm{EX}=260 \mathrm{~nm}$ and $\mathrm{EM}=\sim 440 \mathrm{~nm}$ is an example of these background effects. This feature, as well as the Rayleigh and Raman scattering features, are seen clearly in Figure 1 (HPLC-grade water blank). Given the age of the Schweppe library samples, it is expected that the solutions may also include species arising from decomposition of the dyes.

\subsection{Decomposition of the Dyes}

The effect of decomposition can be seen, for example, with the decades-old Schweppe sample of Acid Blue 74 (CI 73015) (nominal mass $466 \mathrm{Da}$ ). Figure 2 compares the $3 \mathrm{D}$ fluorescence spectrum of that solution with that of a solution freshly prepared from commercial reagentgrade dye. The fluorescence intensity as well as the centroid of the principal peak is clearly different in the fresh solution. These differences can be understood by reference to the corresponding MALDI-TOF mass spectra, shown in Figure 3. The mass spectrum of the decomposed sample shows only a small peak at $\mathrm{m} / \mathrm{z} 421$ (the singly-charged molecular ion $[\mathrm{M}-2 \mathrm{Na}+\mathrm{H}]^{-}$compared with the large $\mathrm{m} / \mathrm{z} 421$ peak from the fresh sample.

\subsection{Uniqueness of the 3D Fluorescence Patterns}

Apart from the few Schweppe dyes showing no fluorescence other than background features, the patterns of the fluorescent dyes appear to be unique, even for structurally very similar dyes. The observation of Clarke [3] and of van Bommel, et al. [4], is borne out for some comparisons, such as S5 Acid Yellow 36 (CI 13065) vs S6 Acid Orange 5 (CI 13080) shown in Figure 4: subtle differences in the overall patterns are more diagnostic than a 
Table 1. 3D fluorescence spectra of dyes in the Schweppe Library. Grouped by structure type.

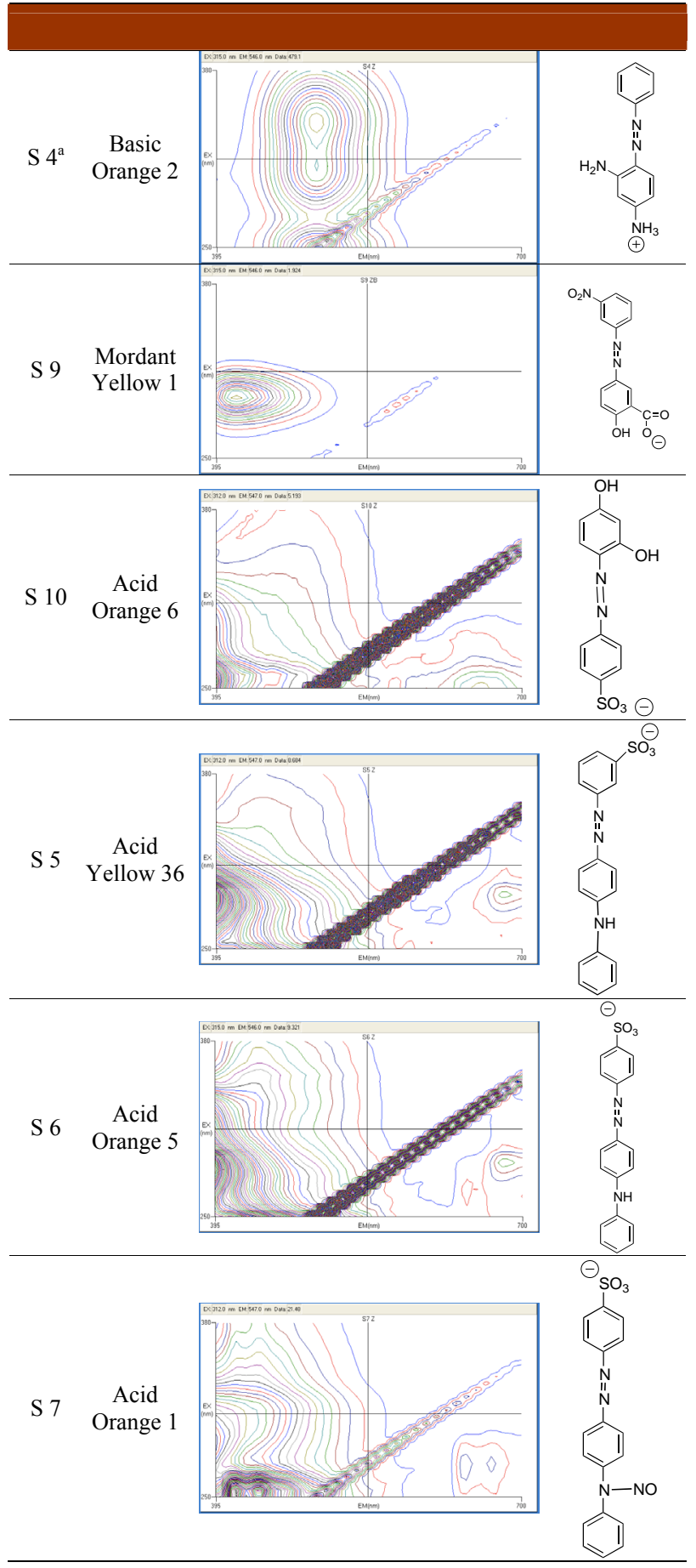

${ }^{a}$ Schweppe Library number.

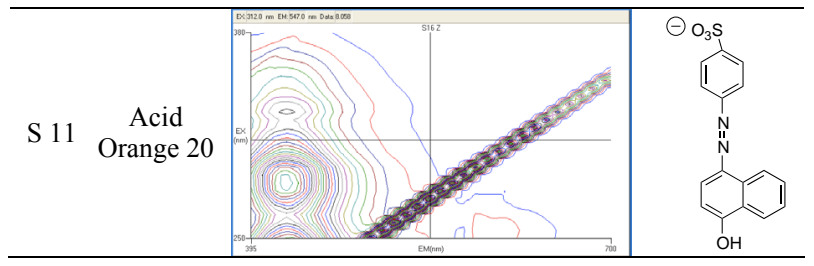

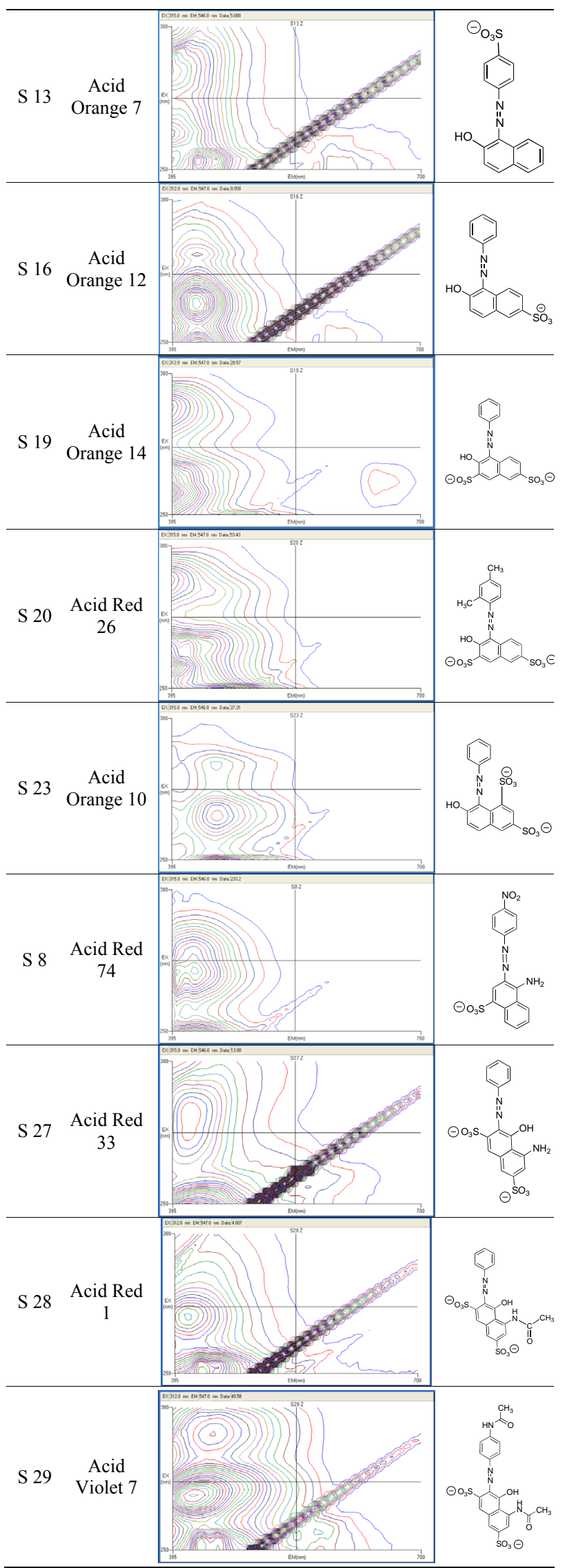



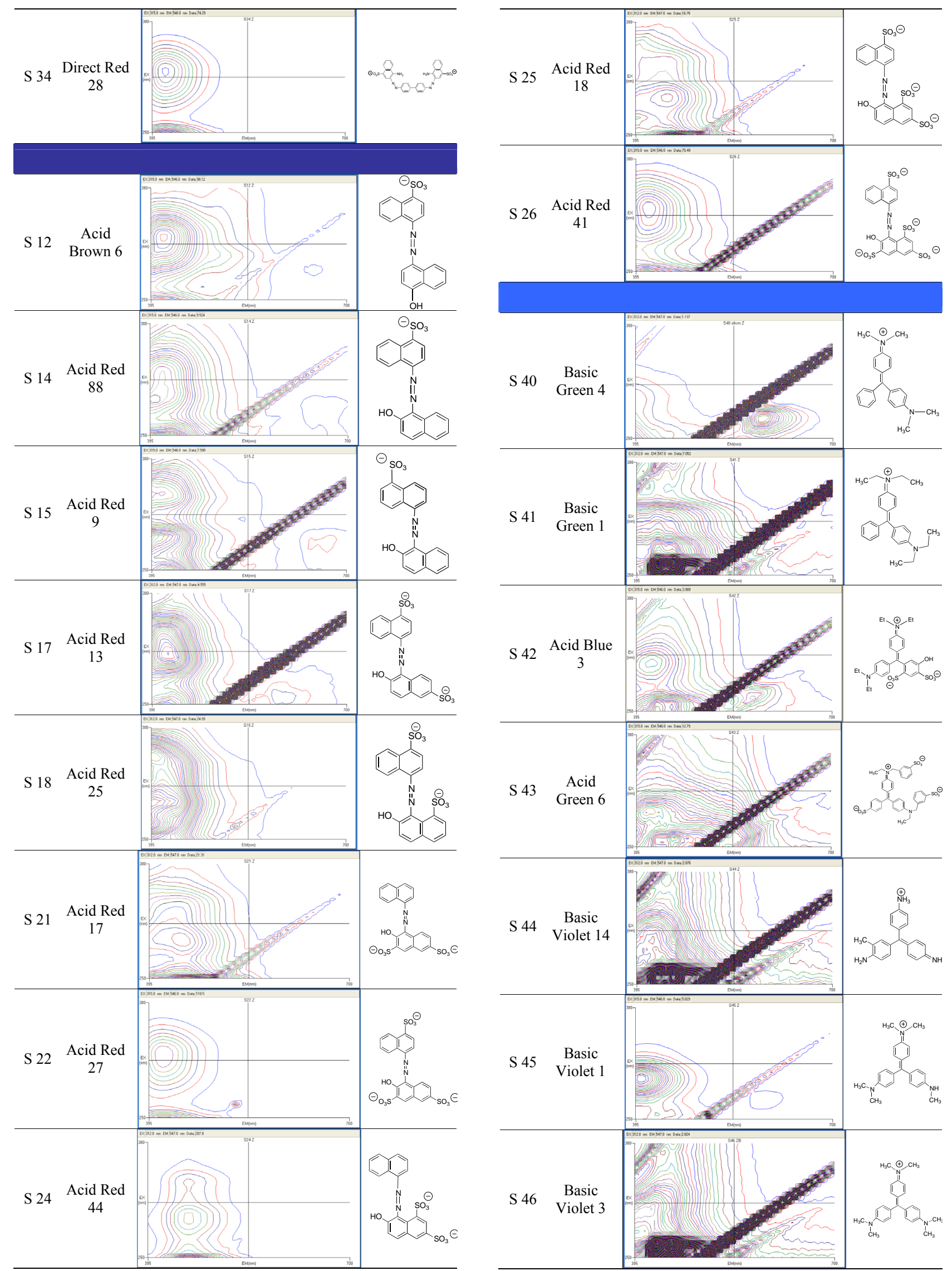

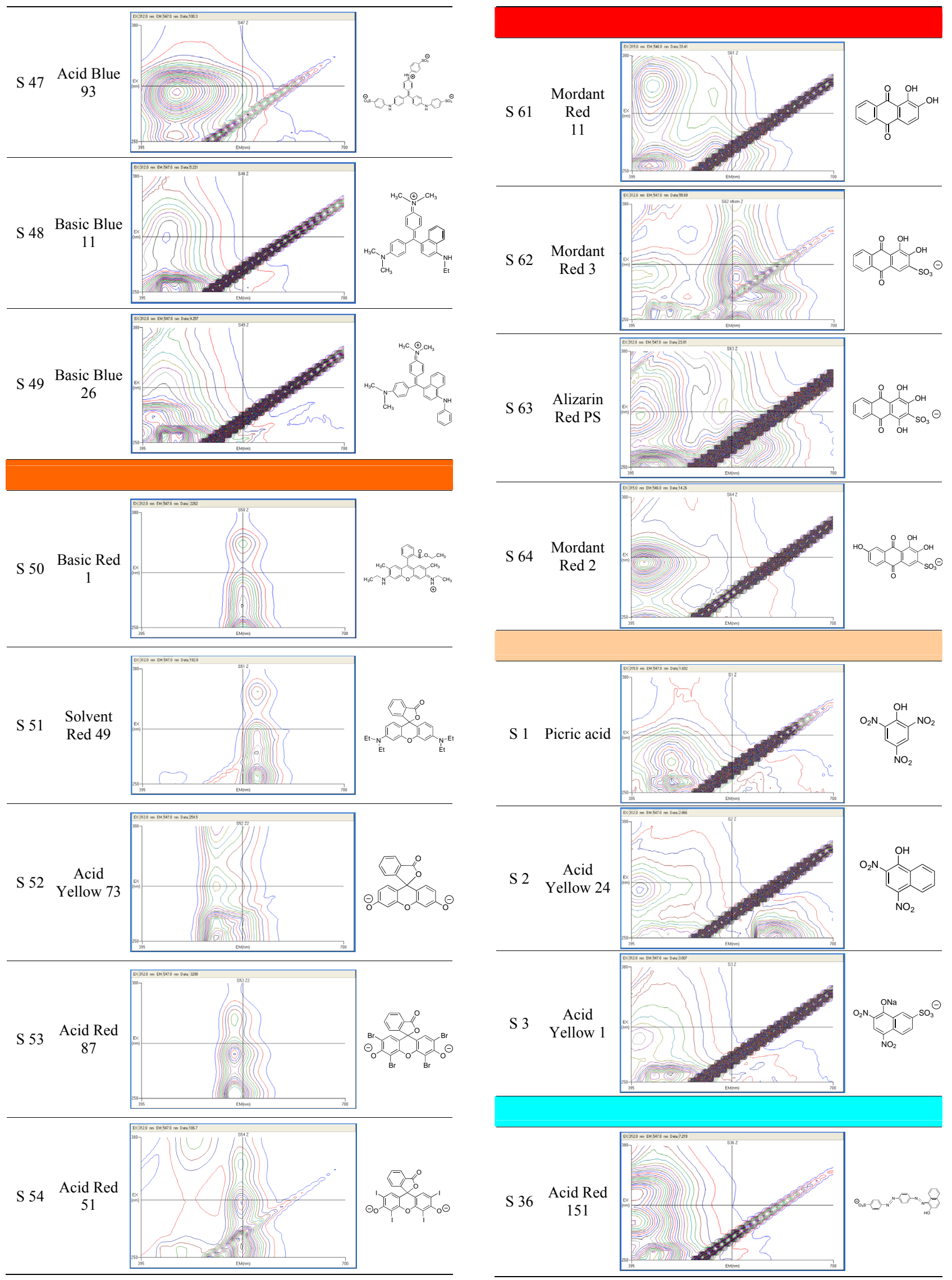


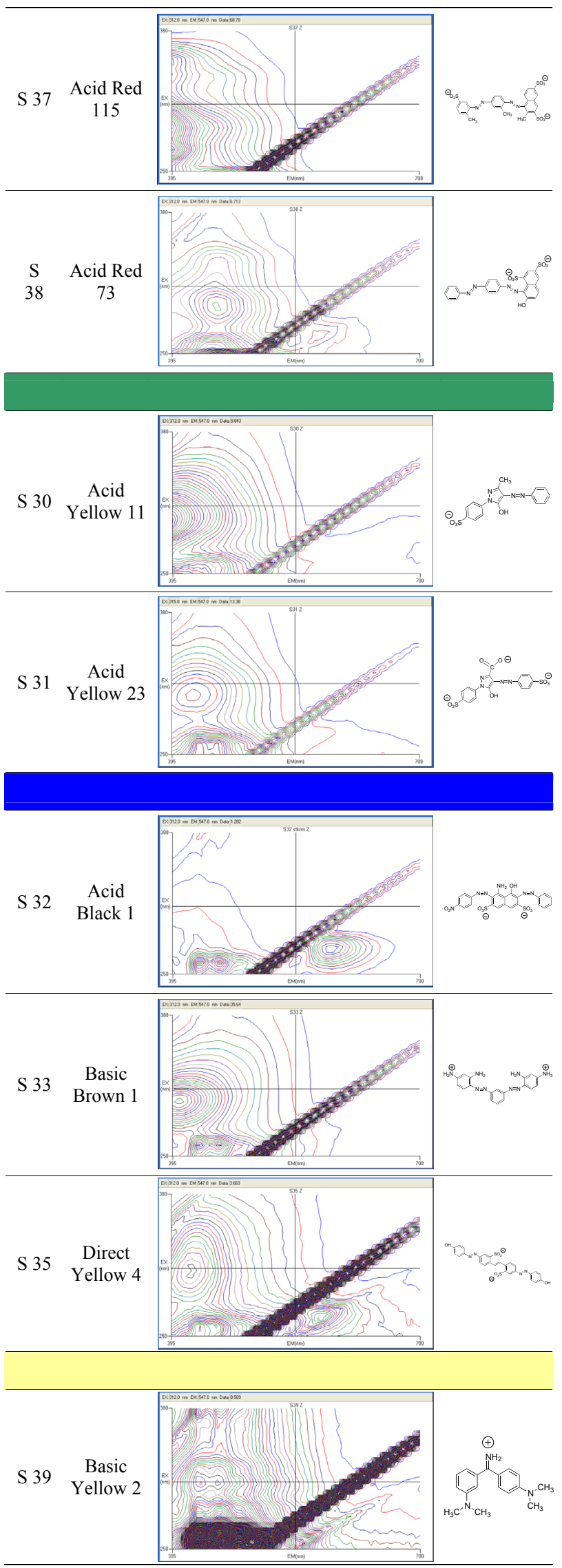

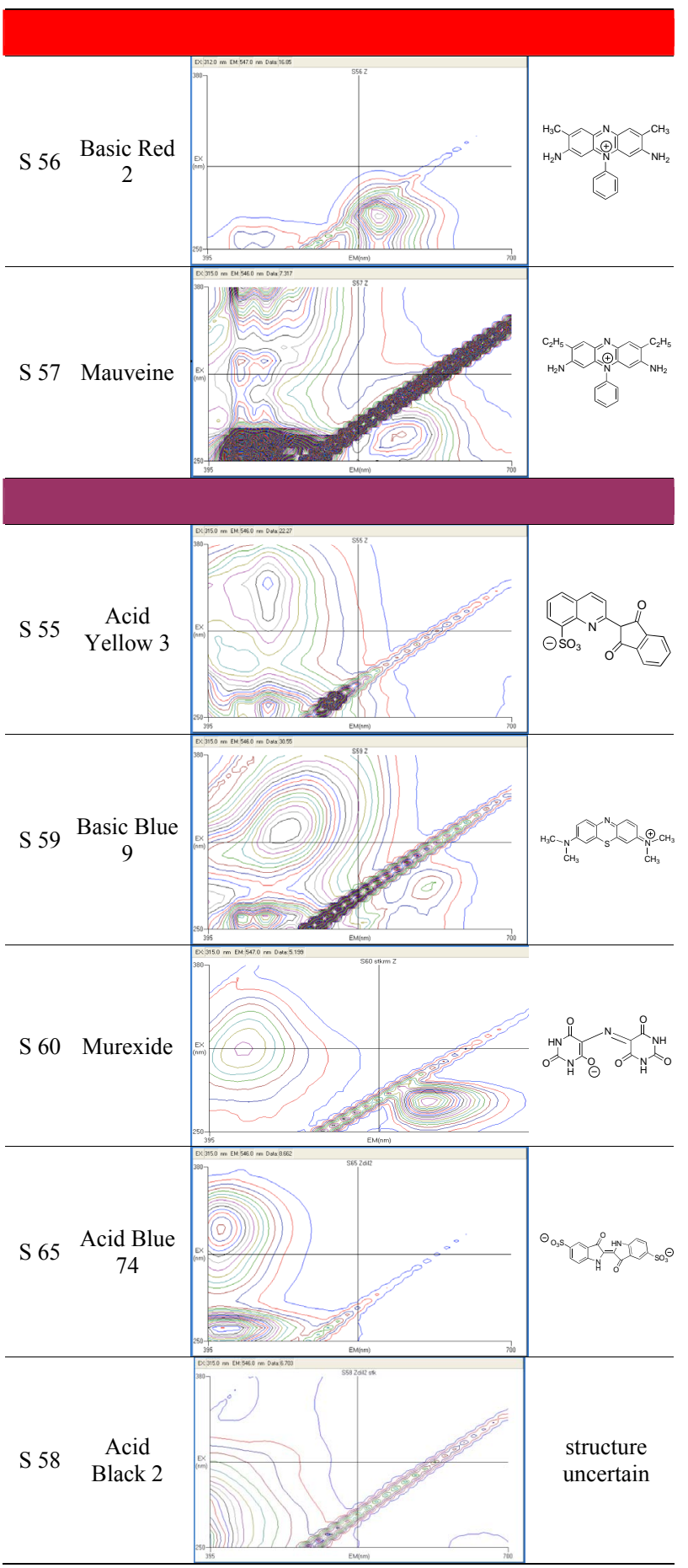

tabulation of peak positions would be. These two isobaric dyes are not distinguished by MALDI-TOF mass spectrometry [2]. Their 3D fluorescence spectra are, indeed, very similar but not identical. If the contour patterns are viewed as topographic maps, one sees a prominent "hill" centered around $\lambda_{\mathrm{EX}} 290 \mathrm{~nm}$ and $\lambda_{\mathrm{EM}} 395 \mathrm{~nm}$ in both spectra. However, the topographic profile of the "descent" from the "summit" toward the "northeast" is noticeably different for the two dyes. 


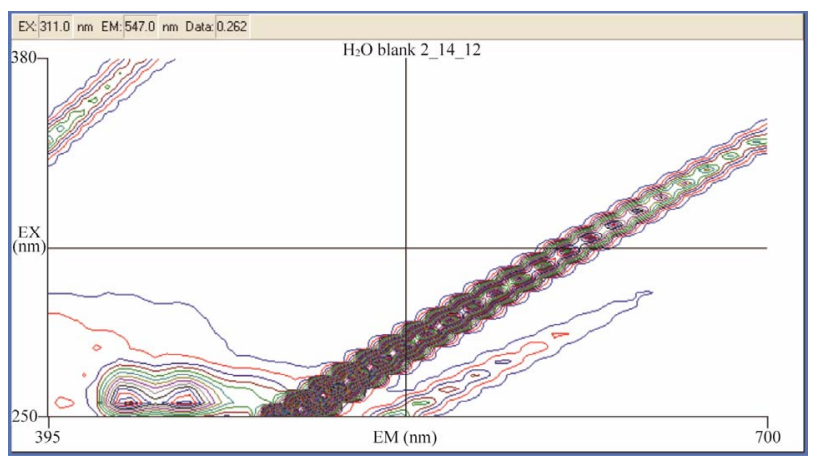

Figure 1. 3D fluorescence spectrum of HPLC grade water showing diagonal features resulting from (left to right) Raman scattering of $1^{\text {st }}$ order Xe lamp photons by water vibration mode $v_{3}$, Rayleigh scattering of 2 nd order Xe lamp photons, and Raman scattering of 2nd order Xe lamp photons by water vibration mode $v_{2}$. The mask-like artifact in the lower left is a transient feature [10].

Table 2. Raman scattering from water seen in 3D fluorescence spectra.

\begin{tabular}{ccc}
\hline Raman shift from water $\nu_{3}$ & $\lambda_{\mathrm{EX}}(\mathrm{nm})$ & $\lambda_{\mathrm{EM}}(\mathrm{nm})$ \\
\cline { 2 - 3 }$\left(\sim 3400 \mathrm{~cm}^{-1}\right)[11]$ & 248 & 271 \\
\hline & 313 & 350 \\
& 365 & 416 \\
& 405 & 469 \\
& 436 & 511 \\
\hline Raman shift from water $v_{2}$ & $\lambda_{\mathrm{EX}}(\mathrm{nm})$ & $\lambda_{\mathrm{EM}}(\mathrm{nm})$ \\
\cline { 2 - 3 }$\left(\sim 1550 \mathrm{~cm}^{-1}\right)^{*}$ & 510 & 553 \\
\hline & 530 & 578 \\
& 550 & 600 \\
& 570 & 625 \\
& 590 & 649 \\
& 610 & 674 \\
\hline
\end{tabular}

"Calculated values.

\subsection{Robustness of the 3D Fluorescence Patterns}

In order to be useful analytically, the 3D fluorescence patterns need to be relatively invariant over the range of conditions likely to be encountered. Some of these dyes are $\mathrm{pH}$ indicators, and we did not attempt to buffer the experimental media. For the majority of the dyes, however, the principal question is whether the $3 \mathrm{D}$ fluorescence patterns are sensitive to concentration. We investigated this issue for several of the dyes in the Schweppe Library. In each case, we prepared a stock solution by weighing out the solid dye with an Ohaus GA200D $0.01 \mathrm{mg}$ balance and dissolving in Aldrich A452 HPLC grade methanol. Spectra were run for several different dilutions of each stock solution. The comparisons show that, aside from small shifts in the centroids of peaks, the patterns are essentially unaffected by concentration. One such comparison is shown in Figure 5.
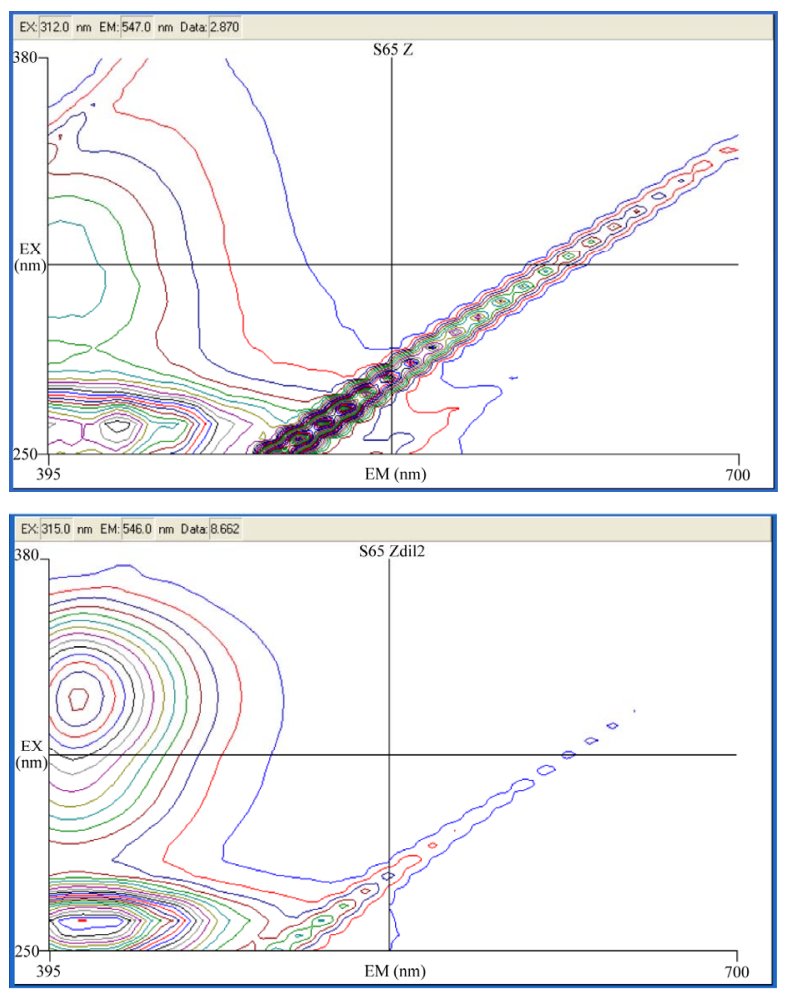

Figure 2. Comparison of 3D fluorescence spectra of original Schweppe sample (top) and fresh commercial sample of Acid Blue 74 (CI 73015) (botttom).
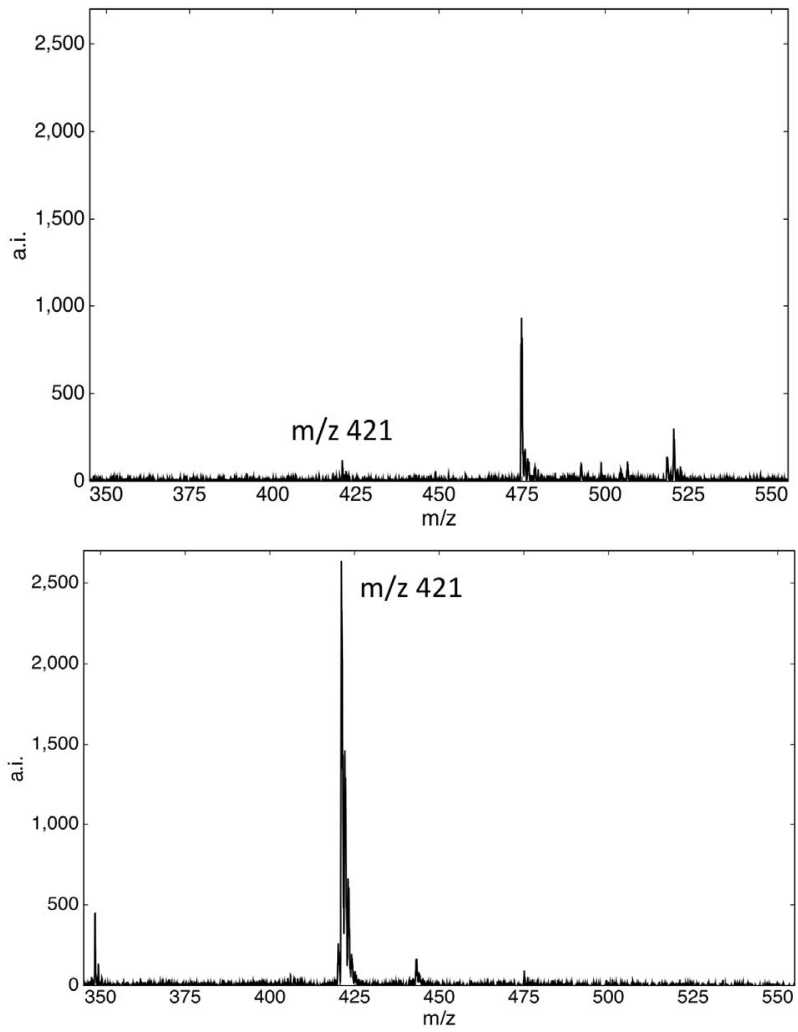

Figure 3. Negative ion MALDI-TOF mass spectra of the two Acid Blue 74 samples shown in Figure 2. 

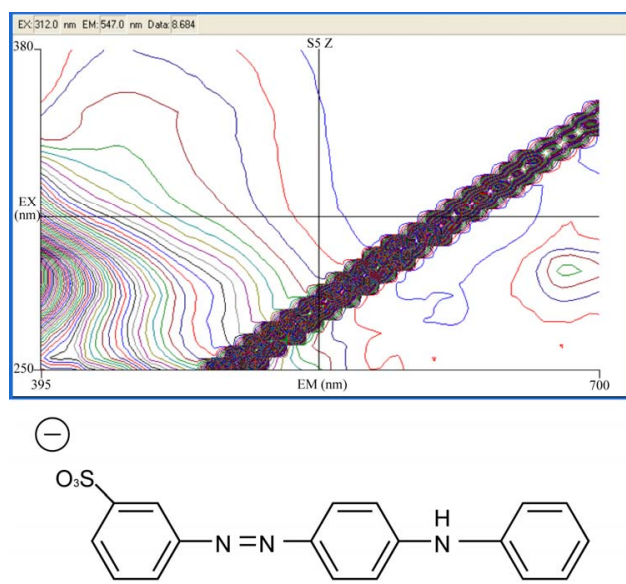
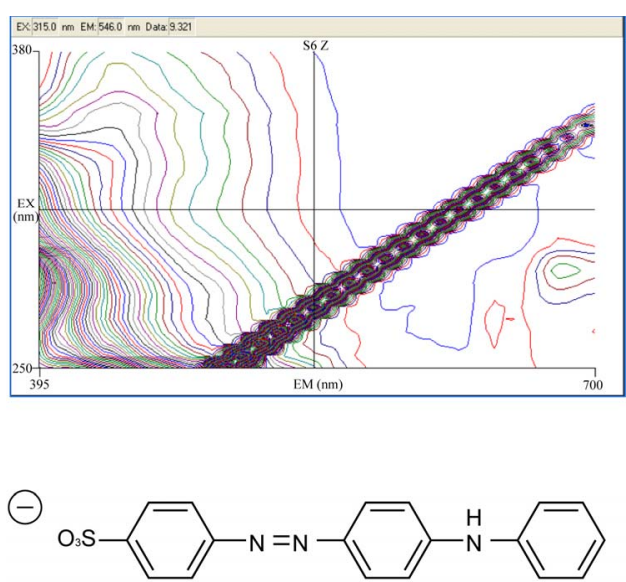

Figure 4. 3D fluorescence spectra of isomeric dyes Acid Yellow 36 (CI 13065) (left) and Acid Orange 5 (CI 13080) (right).

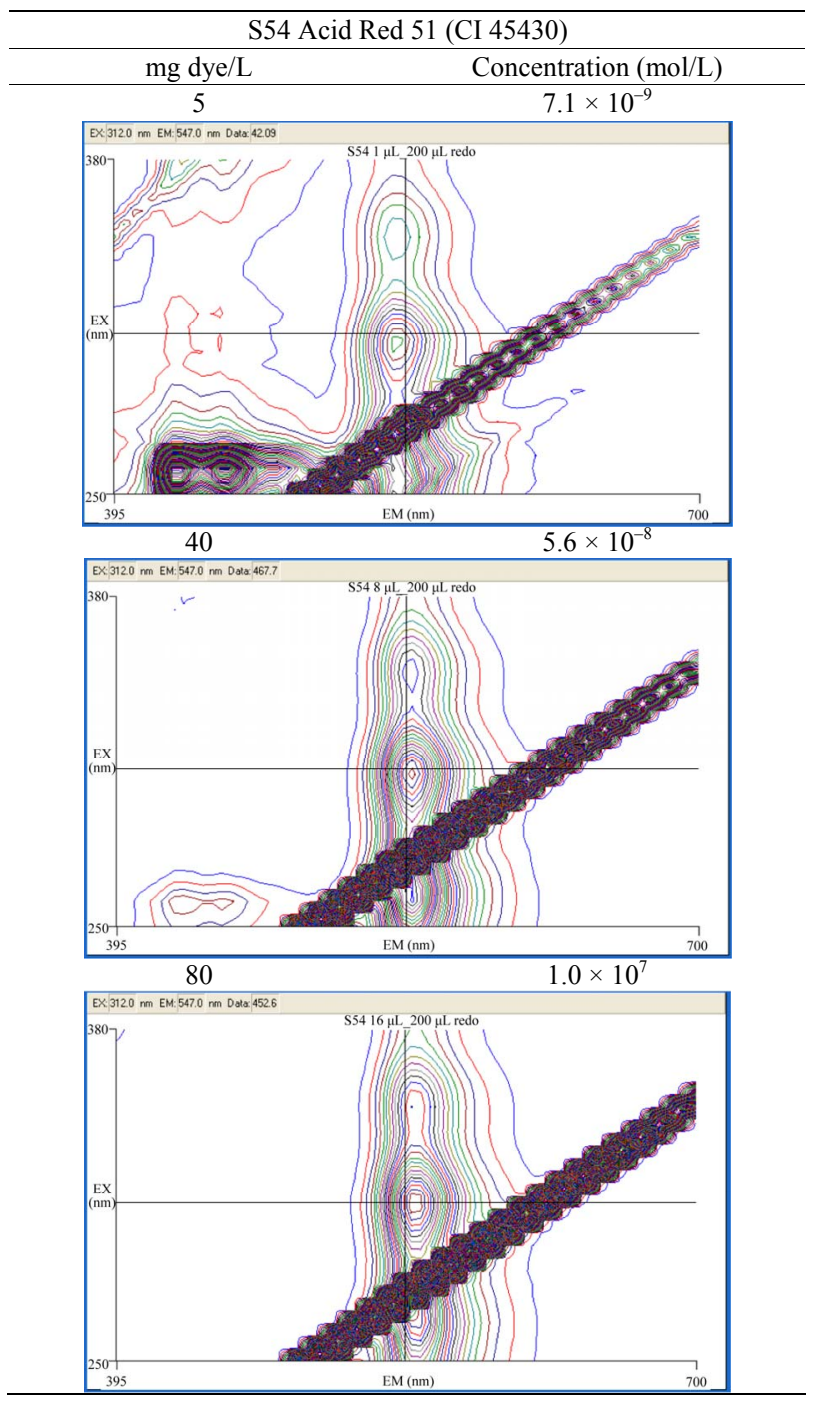

Figure 5. Effect of concentration on 3D fluorescence pattern of Acid Red 51 (CI 45430). Note that the contour interval for the most dilute sample is set to a smaller value than for the other two spectra in order to emphasize the shape of the pattern.

\subsection{Sensitivity}

In a previous study of this same dye library using MALDITOF mass spectrometry, we found a limit of detection on the order of $10^{-13} \mathrm{~mol}$ of dye on the MALDI target [2]. The dye solutions studied here typically correspond to about $10^{-8}$ mol of dye in the cuvette, since the microcell requires only $200 \mu \mathrm{L}$ of solution. Using Acid Red 27 (CI 16185) as an example, the limit of detection for a recognizable $3 \mathrm{D}$ fluorescence pattern is conservatively about 3 $\times 10^{-8} \mathrm{M}$, or $6 \times 10^{-12} \mathrm{~mol}$ of dye in the cuvette. For the highly fluorescent dye Basic Red 1 (CI 45160), the limit of detection is about $3 \times 10^{-9} \mathrm{M}$, or $6 \times 10^{-13} \mathrm{~mol}$ of dye in the cuvette.

\subsection{Application to an Historic Sample}

The development of synthetic dyes following Perkin's 1856 discovery of mauvieine coincided approximately with the opening of Japan to the West, beginning in 1852. It is of interest to ascertain how quickly Japanese artists adopted synthetic dyes for printing inks and other applications. In this context, we extracted a purple dye from a fragment of a Japanese block print from around 1900 (Figure 6). The dye extracted readily with water. The fluorescence spectrum of the dye extract is shown in Figure 7, compared with an extract from unused white drawing paper. There is evidently no fluorescence from the dye solution other than that due to background features. None of the patterns from the Schweppe Library dyes can be recognized in Figure 7.

It has been noted elsewhere that natural dyes are typically not fluorescent, in contrast with most synthetic organic dyes [12]. Knowing that this ink is likely based on natural dyes narrows the field of candidates to the plant or animal sources known to have been used in Asia.

These are relatively few in number $[5,13]$. There are no pure purple dyes among those candidates, so a purple mixture would have been made from madder, safflower, 
or redbud (red) and indigo or dayflower (blue). Figure 8 compares the MALDI-TOF mass spectrum of the purple

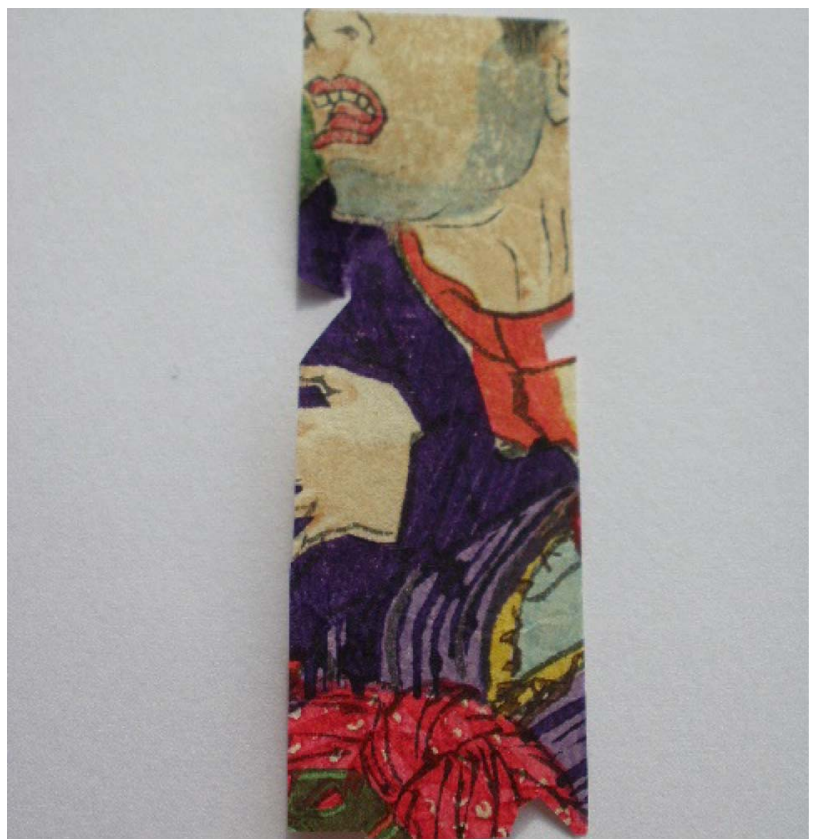

Figure 6. Fragment of early 20th C Japanese block print (height $=58 \mathrm{~mm}$ ). Ink sample analyzed is from purple area of robe.
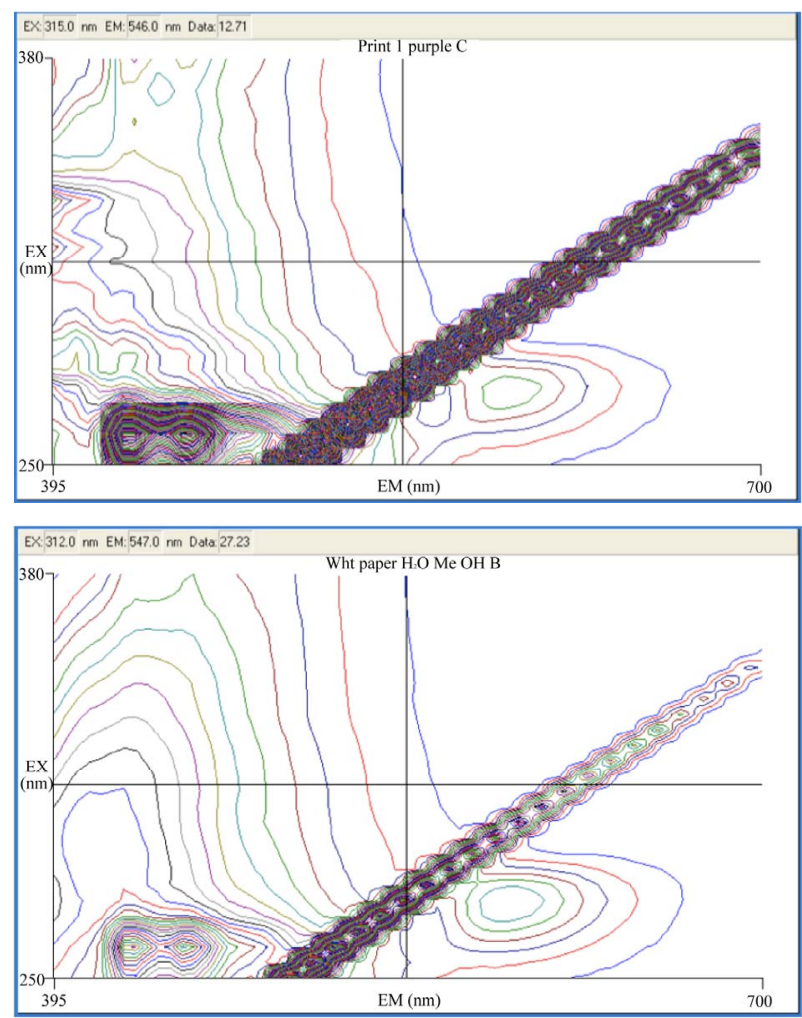

Figure 7. 3D fluorescence spectrum of purple ink (top) from 20th C Japanese block print compared with extract of unprinted drawing paper (bottom).

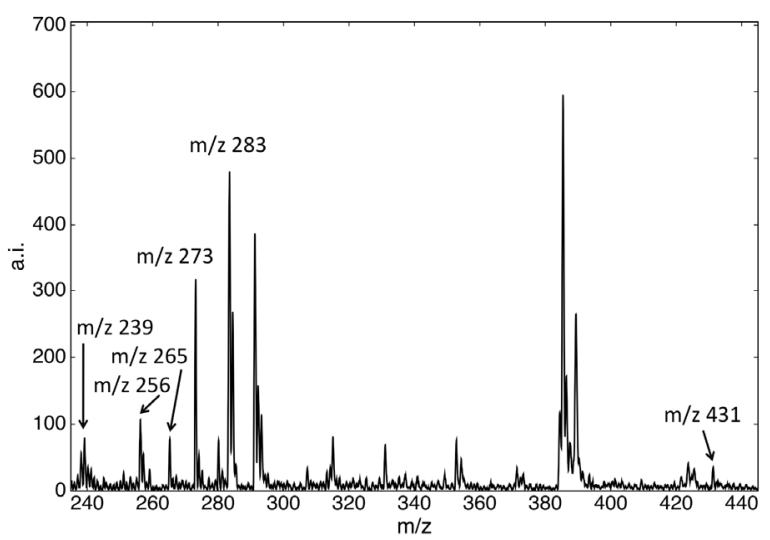

(a)

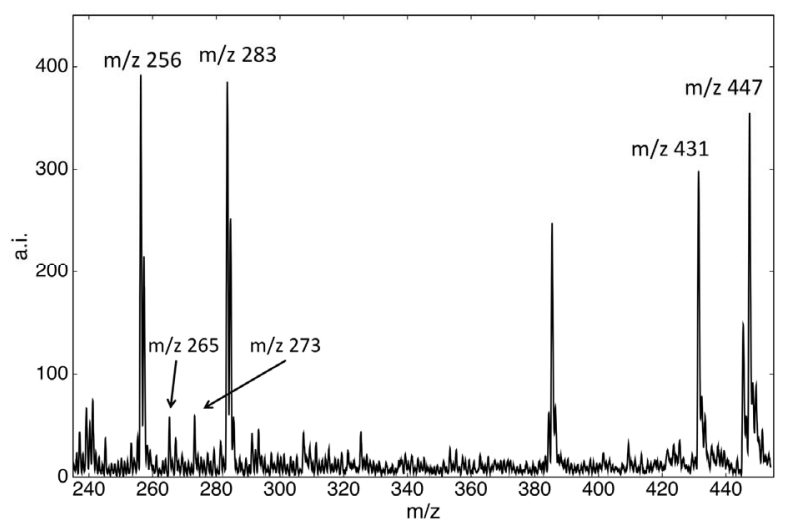

(b)

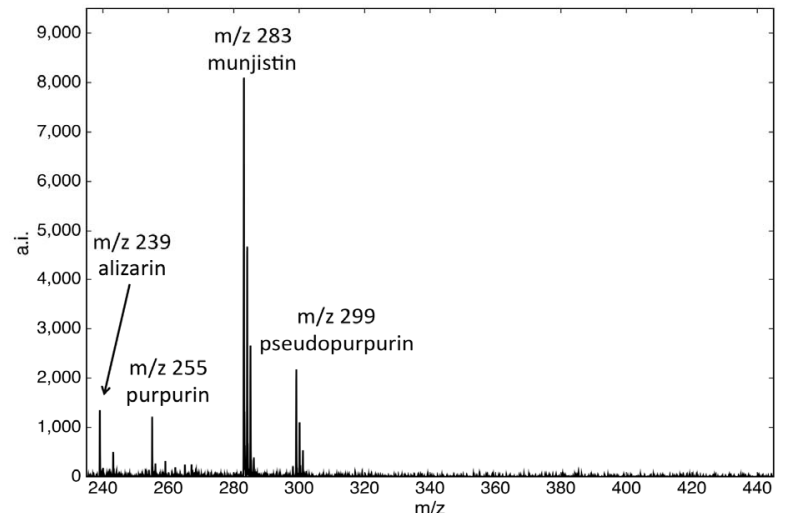

(c)

Figure 8. Negative ion MALDI-TOF mass spectra of (a) purple ink from Japanese block print; (b) blue dye from Commelina communis (dayflower) petal; (c) ground Rubia cordifolia (Indian madder) root.

ink extracted from the print with mass spectra of madder root and dayflower petal extracts. This comparison, taken together with the above observations, suggests that this purple ink was prepared from a mixture of a red madder dye plus the blue dayflower blue dye.

Because of the distinctive fluorescence patterns of the synthetic dyes studied and the high sensitivity of this method, it is very unlikely that this ink sample contains a 
synthetic dye.

\section{Conclusion}

3D fluorescence spectra can provide an important tool for the identification of dyes. These spectra serve as fingerprints that are virtually unique, even among closely related dyes. The sensitivity of fluorescence spectrophotometry means that identification can be accomplished with very small analyte samples. Supplemented by mass spectrometry when necessary, dye identification using 3D fluorescence is a rapid and reliable analytical strategy.

\section{Acknowledgements}

We thank the Camille and Henry Dreyfus Foundation for a Senior Faculty Mentor grant supporting the participation of Lor and Okey-Igwe. We also thank the National Science Foundation for grant CHE-0216268. Mass spectra in this manuscript were prepared using the application mMass [14].

\section{REFERENCES}

[1] Boston Museum of Fine Arts, "Chilkat Dancing Blanket," Museum of Fine Arts, Boston, 2008.

[2] L. J. Soltzberg, A. Hagar, S. Kridaratikorn, A. Mattson and R. Newman, "MALDI-TOF Mass Spectrometric Identification of Dyes and Pigments," Journal of the American Society for Mass Spectrometry, Vol. 18, No. 11, 2007, pp. 2001-2006. doi:10.1016/j.jasms.2007.08.008

[3] M. Clarke, "A New Technique for the Non-Destructive Identification of Organic Pigments, Dyes and Inks in-situ on Early Mediaeval Manuscripts, Using 3-D Fluorescence Reflectance Spectroscopy," Proceedings of the 6th International Conference on Non-Destructive Testing and Microanalysis for the Diagnostics and Conservation of the Cultural and Environmental Heritage ART'99, Rome, May 1999, pp. 1421-1436.

[4] M. R. van Bommel, I. Vanden Berghe, A. M. Wallert, R Boitelle, J. Wouters, "High-Performance Liquid Chromatography and Non-Destructive Three-Dimensional Fluorescence Analysis of Early Synthetic Dyes," Journal of Chromatography A, Vol. 1157, No. 1-2, 2007, pp. 260-272. doi:10.1016/j.chroma.2007.05.017

[5] S. Shimoyama, Y. Noda and S. Kasuhara, "Non-Destructive Analysis of Ukiyo-E Prints," In: P. W. Rogers, Ed., Dyes in History and Archaeology: 15, Textile Research
Associates, York, 1996.

[6] Schweppe Collection of Important Early Synthetic Dyes (Getty Conservation Institute, Los Angeles, CA). "Practical Information for the Identification of Early Synthetic Dyes," Conservation Analytical Laboratory, Smithsonian Institution, Washington DC, 1987.

[7] L. J. Soltzberg, J. D. Slinker, S. Flores-Torres, D. A. Bernards, G. G. Malliaras, H. D. Abruna, J.-S. Kim, R. H. Friend, M. D. Kaplan and V. Goldberg, "Identification of a Quenching Species in Ruthenium Tris-Bipyridine Electroluminescent Devices," Journal of the American Chemical Society, Vol. 128, No. 24, 2006, pp. 7761-7764. doi: $10.1021 / \mathrm{ja} 055782 \mathrm{~g}$

[8] F. J. Green, "The Sigma-Aldrich Handbook of Stains, Dyes and Indicators," Aldrich Chemical Company, Milwaukee, 1990.

[9] T. G. Adiks, A. F. Bunkin, V. A. Luk'yanchenko and S. M. Pershin, "Variation in the Fluorescent Background in Raman Spectra of Distilled Water Purified by Different Methods," Physics of Wave Phenomena, Vol. 16, 2008, pp. 1-6.

[10] L. V. Belovolova, M. V. Glushkov, E. A. Vinogradov, V. A. Babintsev and V. I. Golovanov, "Ultraviolet Fluorescence of Water and Highly Diluted Aqueous Media," Physics of Wave Phenomena, Vol. 17, No. 1, 2009, pp. 21-31. doi:10.3103/S1541308X0901004X

[11] "Hitachi High-Technologies Corporation Fluorescence Spectrophotometer Instruction Manual-FL Solutions Program-Operation," 3rd Edition, Hitachi High-Technologies Corporation, 24-14, Nishi-Shimbashi 1-Chome, Minatoku, Tokyo, 2001.

[12] M. Clarke, "Limitations of Fluorescence Spectroscopy as a Tool for Non-Destructive in situ Identification of Organic Pigments, Dyes and Inks," Presented at 7th International Conference on Non-Destructive Testing and Microanalysis for Diagnostics and Conservation of Cultural and Environmental Heritage, Antwerp, 2-6 June 2002.

[13] R. L. Feller, M. Curran and C. Bailie, "Identification of Traditional Organic Colorants in Japanese Prints and Determination of Their Rates of Fading," In: R. S. Keyes, Ed., Japanese Woodblock Prints: A Catalogue of the Mary A. Ainsworth Collection, Allen Memorial Art Museum, Oberlin College, Oberlin, 1984.

[14] M. Strohalm, M Hassman, B. Košata, M. Kodíček, "mMass Data Miner: An Open Source Alternative for Mass Spectrometric Data Analysis," Rapid Communications in Mass Spectrometry, Vol. 22, No. 6, 2008, pp. 905-908. doi:10.1002/rcm.3444 